\title{
Tissue engineering and regenerative medicine in skin wound healing: what has been done recently -a mini review
}

\begin{abstract}
Many approaches in Tissue Engineering and Regenerative Medicine for skin alternatives have developed models using skin cells and biomaterial scaffolds. There are still many counteracting factors to produce skin alternatives for clinical applications and this mini review shows some human skin models recently developed and the perspective for future use.
\end{abstract}

Keywords: tissue engineering, regenerative medicine, skin, wound, healing
Volume 2 Issue 4 - 2017

\author{
Claudia Mirian de Godoy Marques,' Daniela \\ Ota Hisayasu Suzuki, Jefferson Luiz Brum \\ Marques $^{2}$ \\ 'Department of Health Sciences, State University of Santa \\ Catarina, Brazil \\ ${ }^{2}$ Department of Electrical and Electronic Engineering, Federal \\ University of Santa Catarina, Brazil
}

\begin{abstract}
Correspondence: Claudia Mirian de Godoy Marques, Department of Health Sciences, CEFID-UDESC, Rua Pascoal Simone, 358, Coqueiros, Florianópolis (SC), 88080-350, Brazil, Tel (55-48) 3664-8642, Email claudia.marques@udesc.br
\end{abstract}

Received: May 18, 2017| Published: June 15, 2017

\section{Introduction}

Humans among other animals are made of tissue cells and the fragility of the organism reveals the need of healing when wounded. Since the beginning of Tissue Engineering (TE) very well described by Meyer et al., ${ }^{1}$ there have been many attempts to replace, reconstruct, regenerate and heal body tissues, organs and members which were lost provoked by surgery, burns, infection and birth defect. Tissue lack or dysfunction may turn out to be life threatening and a serious health problem due to shortage of tissues and organ donors.

TE applies different methods to rebuild a new functioning tissue for replacement of its lost body part. Advantages on this approach are the utilization of patient's own tissues, reducing rejection, corticoids and immunosuppressors administration. Animal work may not be needed as human cells and scaffolds of human or biomaterials are utilized. The drawbacks are still long-term patient's tissue lab production and, sterilization of tissues ready for implantation. For new-engineered tissues, vascularization is still a challenge although some body tissues are not vascularised such as cartilage and epithelium. Other difficulties are neuron connection and control of cell metabolic activity.

Although there are still some challenges in solving tissue engineered skin problems such as vascularisation and porous size for cell attachment, new techniques and methodologies are created in order to address these counteracting challenges. The development of smart scaffolds used for seeding cells to proliferate and differentiate making a new tissue turns out a feasible building of a biological structure showing similar morphology and efficiency in function.

Regenerative Medicine (RM) emerged as a result of TE in which stem cells are used with the same aim to replace lost tissues or organs. Regenerative Medicine shows some difficulties as cell differentiation may be unpredictable, the reduced similarities to original tissue organ morphology and, the most serious problem of neoplasic turning over of stem cells resulting in a complex health problem making it difficult to find treatment and resolution for a clinical patient.

Based on the need of stem cells for wound healing, mesenchymal stem cells can be used as mode of action during regenerative medicine and healing process as well as their application in chronic wounds treatment. ${ }^{2}$ In respect to chronic wounds as depleted region of stem cells, indicates a prospectus field for studying better therapies and strategies to be developed utilizing epidermal stem cells and other adult stem cells as tools for cells and scaffolds based therapies for non-healing wounds and other skin disorders. ${ }^{3-5}$

The aim of this mini review was to find out which scaffold constructs together with cells lines have been used in in vitro studies to project new and better skin substitutes to be introduced in clinical approaches and improve human skin when tegument replacement is mandatory in health.

\section{Method}

It was searched in the literature articles during a period of approximately last two years to verify what has been done in Tissue Engineering and Regenerative Medicine of skin considering in vitro studies with clinical perspectives. Using a literature search of MEDLINE/PubMed databases, research studies were limited to those published in the period of 1st January 2015 to 17th April 2017 and limited to humans and in vitro work. Review articles were excluded from our quest. Searching for the term "tissue engineeringregenerative medicine - skin wound healing", it was found 27 articles which only 9 were selected as matched to the aim of the review.

From these 9 articles, only 6 reported tissue engineering or regenerative medicine in human skin wound healing, considering the composition of the scaffold, cell lines used and the purpose of its use in healing and/or regeneration. 


\section{Results and discussion}

Table 1 shows many types of scaffolds and cells used in vitro with the same general objective of skin wound healing and regeneration. With one exception, mouse embryonic fibroblasts cells were used whilst other studies seeded human cells on scaffolds. Scaffolds fabrication varied from common biomaterials such as collagen, cryogels generated from acrylated hyaluronan and, autologous skin micro-graft to hybrid scaffolds such as human amniotic membrane together with silk fibroin nanofibers. In just one article some scaffold types were used such as chitosan, fibrin, bovine collagen and decellularized porcine dermis.

Table I Scaffolds and cells used in TE and RM for skin wound healing.

\begin{tabular}{|c|c|c|c|}
\hline References & Biomaterial/scaffold & Cell type & Objective \\
\hline 6 & $\begin{array}{l}\text { Cryogels generated from acrylated hyaluronan } \\
\text { (polymerization initiated by accelerated electrons) }\end{array}$ & Human dermal fibroblasts & Wound healing skin \\
\hline 7 & $\begin{array}{l}\text { Autologous micro-grafts from cutaneous tissue- and collagens } \\
\text { sponges }\end{array}$ & $\begin{array}{l}\text { Skin cells and, mesenchymal stem cells } \\
\text { (from other tissues) }\end{array}$ & $\begin{array}{l}\text { Acute and chronic skin } \\
\text { lesions }\end{array}$ \\
\hline 8 & Collagen (careful process of collagen scaffolds) & Myofibroblasts & Skin wounds and \\
\hline 9 & Chitosan, fibrin, bovine collagen, decellularized porcine dermis & $\begin{array}{l}\text { Human adipose-derived mesenchymal } \\
\text { stem cells }\end{array}$ & Dermal wounds \\
\hline 10 & $\begin{array}{l}\text { Combined human amniotic membrane (HAM) and silk fibroin } \\
\text { nano-fibers to produce a bilayer construct }\end{array}$ & Mouse embryonic fibroblasts & Skin regeneration \\
\hline II & Platelet lysate incorporated in collagen as gel beads & Human adipose-derived stem cells & Skin ulcers treatment \\
\hline
\end{tabular}

According to present reviewed studies, cell seeding were performed as expected adding dermal fibroblasts, skin cells, mesenchymal stem cells, myofibroblasts and human adipose-derived stem cells to a variety of described scaffolds.

Each study demonstrated a great potential in developing methodologies and strategies to produce the most appropriated tissue engineered construct for future clinical use. There are still some parameters to be defined for a better standardization of skin tissue engineered construct according to the clinical aspect of the wound, tissue/organ and patient profile.

The remaining articles included studies utilizing biomaterials implants for skin and mucosa reconstruction in help for healing and repair. One study ${ }^{12}$ mentioned human amniotic membrane (HAM) revealed a stable wound healing matrix and demonstrated as a useful biomaterial for oral mucosa reconstruction.

A study using mice ${ }^{13}$ demonstrated alternative therapies of human adipose-derived stem cell spheroids in a stem cell-depleted condition such as diabetic chronic skin ulcer. Another interesting study ${ }^{14}$ suggested dextran-based hydrogels as treatment for third degree burn wound healing for rapid wound closure, re-epithelialization, enhanced extracellular matrix remodelling and even nerve reinnervation. If this could be transferred to humans, it could improve extensive burn care and improve healing reducing pain and risk of infection. Other engineered substitutes added adipose-derived stromal cells to temporary wound dressings as potential therapy to promote cutaneous healing in a murine model. ${ }^{15}$

There are some concerns related to in vitro wound healing studies in which the wound dimension such as size and depth of wounds that are not always standardised. Making a pattern of wounds therefore may have a better result in controlled wound healing investigations. The development of an automated wounding device, which generated standardised wounds for healing process measurement under controlled conditions, was described by Rossi et al. ${ }^{16}$ As this method makes possible its use for in vitro studies, it is also carried out under sterilised conditions, ensuring better reproducibility.

There are two articles that could be included in this discussion considering the utilization of biomaterials in TE with the purpose of tissue regeneration and skin wound healing. One ${ }^{17}$ reported the importance of extracellular matrix as a promising approach for tissue engineering. Extracellular matrix scaffolds are able to create a regenerative microenvironment to repair and functioning as a template for various tissues such as skin, bone, nerve, heart, liver and other tissues. Another ${ }^{18}$ was a survey study on application of natural, synthetic and composite polymers to produce electrospun scaffolds as skin substitutes and dressings that demonstrated a promising pathway of skin wound healing and regeneration.

The beauty of TE and RM altogether is not only the production of biological tissues and organs with a good internal control but also in the painstaking methods developed to obtain new replacement options presenting similar morphology and function to the original tissue as well as its esthetical appearance of internal and external body parts replacements.

\section{Conclusion and future prospects}

A variety of scaffolds made of biomaterials and human skin and stem cells have been developed for skin wound healing and regeneration. Despite of many challenges faced by scientists, better approaches in developing tissue engineered skin substitutes and production of new functioning skin tissues by the regenerative medicine are progressively suggesting their clinical application to humans in solving health problems.

\section{Acknowledgements}

None.

\section{Conflict of interest}

The author declares no conflict of interest. 


\section{References}

1. Meyer $U$. The history of tissue engineering and regenerative medicine in perspective. In: Meyer U, et al. Editors. Fundamentals of Tissue Engineering and Regenerative Medicine. 1st ed. New York, USA: Springer-Verlag Berlin Heidelberger; 2009. p. 5-12.

2. Zahorec P, Koller J, Danisovic L, et al. Mesenchymal stem cells for chronic wounds therapy. Cell Tissue Bank. 2015;16(1):19-26.

3. Ojeh N, Pastar I, Tomic Canic M, et al. Stem cells in skin regeneration, wound healing, and their clinical applications. Int $J$ Mol Sci. 2015;16(10):25476-254501.

4. Chen Z, Wang Y, Shi C. Therapeutic implications of newly identified stem cell populations from the skin dermis. Cell Transplant. 2015;24(8):1405-1422.

5. Vapniarsky N, Arzi B, Hu JC, et al. Concise review: human dermis as an autologous source of stem Cells for tissue engineering and regenerative medicine. Stem Cells Transl Med. 2015;4(10):1187-1198.

6. Thönes S, Kutz LM, Oehmichen S, et al. New E-beam-initiated hyaluronan acrylate cryogels support growth and matrix deposition by dermal fibroblasts. Int J Biol Macromol. 2017;94(Pt A):611-620.

7. Purpura V, Bondioli E, Graziano A, et al. Tissue characterization after a new disaggregation method for skin micro-grafts generation. J Vis Exp. 2016;4(109):e53579.

8. Yannas IV, Tzeranis D, So PT. Surface biology of collagen scaffold explains blocking of wound contraction and regeneration of skin and peripheral nerves. Biomed Mater. 2015;11(1):e014106.

9. Wahl EA, Fierro FA, Peavy TR, et al. In Vitro evaluation of scaffolds for the delivery of mesenchymal stem cells to wounds. Biomed Res Int. 2015;2015:e108571.
10. Arasteh S, Kazemnejad S, Khanjani S, et al. Fabrication and characterization of nano-fibrous bilayer composite for skin regeneration application. Methods. 2015;99:3-12.

11. Lima AC, Mano JF, Concheiro A, et al. Fast and mil strategy, using super hydrophobic surfaces, to produce collagen/platelet lysate gel beads for skin regeneration. Stem Cell Rev. 2015;11(1):161-179.

12. Qi F, Yoshida T, Koike T, et al. Construction and characterization of human oral mucosa equivalent using hyper-dry amniotic membrane as a matrix. Arch Oral Biol. 2016;65:26-34.

13. Mineda K, Feng J, Ishimine H, et al. Therapeutic potential of human adipose-derived stem/stromal cell microspheroids prepared by three-dimensional culture in non-cross-linked hyaluronic acid gel. Stem Cells Transl Med. 2015;4(12):1511-1522.

14. Shen YI, Song HH, Papa AE, et al. Acellular hydrogels for regenerative burn wound healing: translation from a porcine model. $J$ invest Dermatol. 2015;135(10):2519-2529.

15. Morissete Martin P, Maux A, Laterreur V, et al. Enhancing repair of full-thickness excisional wounds in a murine model: Impact of tissue-engineered biological dressings featuring human differentiated adipocytes. Acta Biomater. 2015;22:39-49.

16. Rossi A, Appelt Menzel A, Kurdyn S, et al. Generation of a three-dimensional full thickness skin equivalent and automated wounding. J Vis Exp. 2015;96:e52576

17. Yi S, Ding F, Gong L, Gu X. Extracellular matrix scaffolds for tissue engineering and regenerative medicine. Curr Stem Cell Res Ther. 2107; 12(3):233-246.

18. Norouzi M, Boroujeni SM, Omidvarkordshouli N, et al. Advances in skin regeneration: application of electrospun scaffolds. Adv Healthc Mater. 2015;4(8):1114-1133. 